International Journal of Pure and Applied Mathematics

Volume 108 No. 2 2016, 425-450

ISSN: 1311-8080 (printed version); ISSN: 1314-3395 (on-line version)

url: http://www.ijpam.eu

doi: 10.12732 /ijpam.v108i2.17

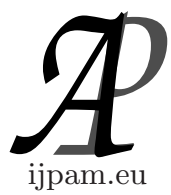

\title{
INFLUENCE OF AN INTERNAL HEAT SOURCE OR SINK ON THE MAGNETOCONVECTION OF A MICROPOLAR FLUID IN A VERTICAL CHANNEL
}

\author{
A. Borrelli ${ }^{1}$, G. Giantesio ${ }^{2}$, M.C. Patria ${ }^{3}$ \\ ${ }^{1,3}$ Dipartimento di Matematica e Informatica \\ Università di Ferrara \\ via Machiavelli 30, 44121 Ferrara, ITALY \\ ${ }^{2}$ Dipartimento di Matematica e Fisica \\ Università Cattolica del Sacro Cuore \\ via Musei 41, 25121 Brescia, ITALY
}

\begin{abstract}
This work examines the effects of an external uniform magnetic field and of an internal heat source or sink on the steady mixed convection in the fully developed flow of a micropolar fluid filling a vertical channel under the Oberbeck-Boussinesq approximation. The two boundaries are kept at different uniform temperatures. The velocity, the microrotation, the temperature and the induced magnetic field are analytically obtained. A selected set of pictures and Tables are reported in order to illustrate the influence of the internal heat parameter on the trend of the motion and in particular on the reverse flow.
\end{abstract}

AMS Subject Classification: 76D10, 76W05, 80A20

Key Words: micropolar fluids, MHD fully developed flow, mixed convection, Boussinesq approximation, vertical channel, heat source/sink

Received: February 22, 2106

Published: June 23, 2016

$\S_{\text {Correspondence author }}$ (c) 2016 Academic Publications, Ltd. url: www.acadpubl.eu 


\section{Nomenclature}

C

$c_{0}, c_{d}, c_{a}$

$2 d$

E

$\mathbf{g}=-g \mathbf{e}_{\mathbf{1}}$

$\mathrm{Gr}$

$\mathbf{H}$

$h(y)$

\section{$H_{0} \mathbf{e}_{2}$}

$H_{1}\left(x_{2}\right)$

I

$k$

$k_{1,2}$

$l$

L

$M^{2}$

$M_{p}^{2}$

$N^{2}$

$\mathrm{Nu}$

$p$

$P=p+\mu_{e} \frac{H_{1}^{2}}{2}+\rho_{0} g x_{1}$

$p_{0}$

Q

$r^{2}$

Re

$T=T\left(x_{2}\right)$ constant such that $P=-C x_{1}+p_{0}$

angular viscosity coefficients

channel width

electric field

gravity acceleration

Grashof number defined by $(5)_{9}$

total magnetic field

dimensionless function describing the induced magnetic

field defined by $(5)_{15}$

external uniform magnetic field $\left(H_{0}>0\right)$

induced magnetic field component in the $x_{1}$-direction

microinertia coefficient

fluid thermal conductivity

heat transfer coefficients evaluated at $\Pi_{1,2}$

characteristic length defined by $(5)_{2}$

dimensionless constant defined by $(5)_{3}$

Hartmann number defined by $(5)_{5}$

micropolar parameter defined by $(5)_{4}$

coupling number defined by $(5)_{1}\left(0<N^{2}<1\right)$

Nusselt number

pressure

difference between the hydromagnetic pressure and the hydrostatic pressure

arbitrary constant

constant rate of volumetric internal heat generation $(Q>0)$

internal heat parameter defined by $(5)_{16}$

Reynolds number defined by $(5)_{8}$

temperature 


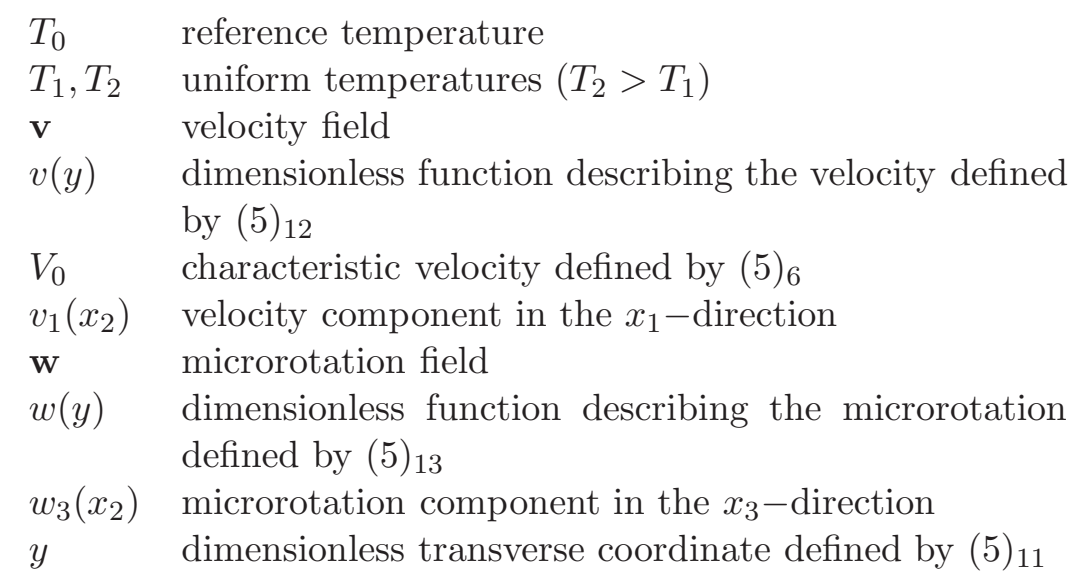

\section{Greek symbols}

$\alpha, \beta, \gamma$ dimensionless constants defined by (10)

$\alpha_{T} \quad$ thermal expansion coefficient

$\eta_{e} \quad$ magnetic diffusivity $\left(\eta_{e}=\frac{1}{\mu_{e} \sigma_{e}}\right)$

$\vartheta(y) \quad$ dimensionless temperature defined by $(5)_{14}$

$\lambda \quad$ buoyancy coefficient defined by $(5)_{10}$

$\mu \quad$ Newtonian viscosity coefficient $(\mu>0)$

$\mu_{e} \quad$ magnetic permeability

$\mu_{r} \quad$ dynamic microrotation viscosity coefficient

$\nu_{0} \quad$ constant defined by $(5)_{7}$

$\rho_{0} \quad$ mass density at the temperature $T_{0}$

$\sigma_{e} \quad$ electrical conductivity

$\tau_{1,2} \quad$ skin friction at the plates $\Pi_{1,2}$

$\boldsymbol{\tau}_{p 1,2} \quad$ skin couple friction at the plates $\Pi_{1,2}$

\section{Introduction}

The aim of this paper is to extend the results obtained in [1] when an internal heat source or sink is present. More precisely, we study the mixed magnetoconvection in the fully developed flow of a micropolar fluid filling a vertical channel under the Oberbeck-Boussinesq approximation ([2]). About the theory of micropolar fluids we refer to [3], [4] and [5].

The magnetoconvecion in a channel has many relevant applications: geothermal reservoirs, cooling of nuclear reactors, electric transmission cables, thermal insulation, electronic chips, semiconductor wafers, etc. 
In our study we solve the problem of the steady mixed convection of a Boussinesquian electrically conducting micropolar fluid in a vertical channel under the action of an external uniform magnetic field normal to the direction of the velocity and to the channel. The walls are kept at constant temperatures $T_{1}$ and $T_{2}\left(T_{1}<T_{2}\right)$. The internal heat source or sink here considered depends only on the temperature difference $T-T_{0}$ and so it is not known a priori. This kind of source/sink occurs in exothermic/endothermic chemical and biochemical processes. There are many papers in the literature concerning such internal heat generation or absorption in several different physical situations ([6], [7], [8], etc.).

The first paper on the fully developed free convection of a micropolar fluid in a vertical channel is [9]; this work has been generalized in [10] in order to consider also the mass transfer. In [11], [12] mixed convection flow with symmetric and asymmetric heating is examined in the presence of heat source-sink.

As far as the influence of an external magnetic field on the convective flow of a micropolar fluid in a vertical channel is concerned, the most exhaustive paper is [1], where there is studied the case in the absence of an internal heat source/sink.

In the present paper we neglect the dissipation terms in the energy equation, as it is usual in the Oberbeck-Boussinesq approximation ([13]), so that we obtain the explicit solution of the problem which takes into account the induced magnetic field. We point out that the induced magnetic field is neglected in most of the works concerning the convective flow in a vertical channel, also in the simpler case of a Newtonian fluid.

The paper is organized in this way:

In Section 2 we formulate the problem from the physical point of view and Section 3 is devoted to solve it in the presence of an internal heat source. We first determine the temperature by solving a boundary value problem which is well-set only if the internal heat generation parameter $r^{2}$ does not take particular values. Then, in order to find the analytical expression of the velocity, microrotation and induced magnetic field, we have to examine three cases (as in [1]) related to the strength of the external uniform magnetic field. Finally, we integrate the boundary value problem which describes the motion in the three cases.

In Section 4 we solve the same problem when an internal heat sink is present. In order to find the analytical expression of the velocity, microrotation and induced magnetic field, we have to distinguish if the square root of the internal heat generation parameter is not a root, is a simple root or is a double root of the characteristic equation and we furnish the solutions in all cases. 
In Section 5 we make some comments about the flow and we compute the Nusselt numbers, the skin and skin couple frictions. We also furnish the flow in the absence of external magnetic field and in the Newtonian case.

In Section 6 we give some pictures in order to show the influence of $r^{2}$ on the flow and we point out some differences about the trend of the flow between the case of heat generation and the case of heat absorption. We refer to [1] for the complete study of the influence of the other material parameters on the flow; as in [1] and [15], for suitable values of the buoyancy parameter $\lambda$, the reverse flow occurs. Precisely, there exists a critical value $\lambda^{*}$ such that

- $\lambda>0$ : if $\lambda>\lambda^{*}$, then the reverse flow occurs near the coldest wall;

- $\lambda<0$ : if $\lambda<\lambda^{*}$, then the reverse flow occurs near the hottest wall.

The value of $\lambda^{*}$ decreases as $r$ increases if there is a source (the decreasing is very significant when $r=3), \lambda^{*}$ increases as $r$ increases if there is a sink so that we can conclude that the presence of a source (sink) tends to favor (prevent) the occurrence of the reverse flow. As far as we know, the influence of $r^{2}$ on the reverse flow has not been studied before in the literature.

If the buoyancy parameter vanishes (symmetric heating), then the phenomenon of the reverse flow does not appear.

\section{Basic Equations}

In this Section we briefly formulate the problem. The Boussinesquian, electrically conducting micropolar fluid fills the region $\mathcal{S}$ between two infinite rigid, fixed, non-electrically conducting vertical plates $\Pi_{1}, \Pi_{2}$ separated by a distance $2 d$ (Figure 1). We assume the region outside the plane to be a vacuum (free space). The coordinate axes are fixed in order to have

$$
\begin{aligned}
& \mathcal{S}=\left\{\left(x_{1}, x_{2}, x_{3}\right) \in \mathbb{R}^{3}:\left(x_{1}, x_{3}\right) \in \mathbb{R}^{2}, x_{2} \in(-d, d)\right\} \\
& \Pi_{i}=\left\{\left(x_{1}, x_{2}, x_{3}\right) \in \mathbb{R}^{3}:\left(x_{1}, x_{3}\right) \in \mathbb{R}^{2}, x_{2}=(-1)^{i} d\right\}, \quad i=1,2
\end{aligned}
$$

and $x_{1}$-axis is vertical upward.

Our aim is to study the steady mixed convection in the fully developed flow of the fluid under the action of an external uniform magnetic field $H_{0} \mathbf{e}_{2}$ normal to planes $\Pi_{1,2}\left(H_{0}>0\right)$ and in the presence of an internal heat source or sink neglecting the viscous and ohmic dissipation terms. 


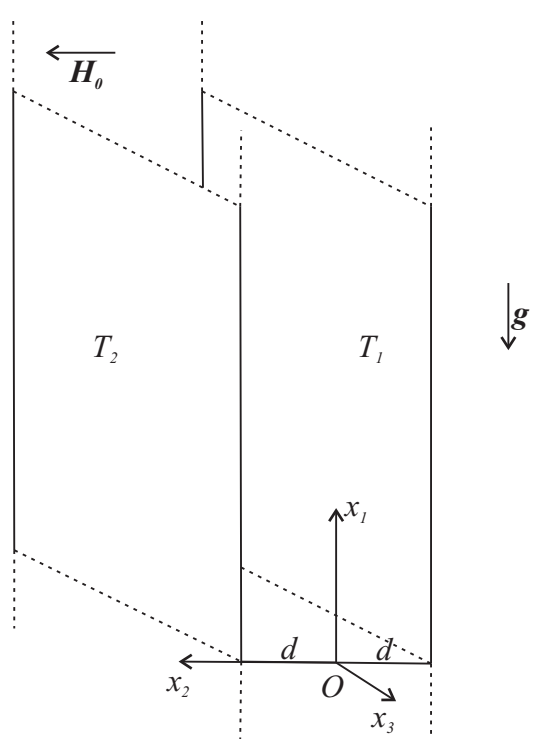

Figure 1: Physical configuration and coordinate system $\left(T_{2}>T_{1}\right)$.

This flow in the absence of external mechanical body forces, body couples and free electric charges under the Oberbeck-Boussinesq approximation is governed by the following equations $([5])$

$$
\begin{aligned}
& \rho_{0} \mathbf{v} \cdot \nabla \mathbf{v}=-\nabla P+\left(\mu+\mu_{r}\right) \triangle \mathbf{v}+2 \mu_{r}(\nabla \times \mathbf{w})+\mu_{e}(\nabla \times \mathbf{H}) \times \mathbf{H} \\
& -\rho_{0} \alpha_{T}\left(T-T_{0}\right) \mathbf{g} \\
& \rho_{0} I \mathbf{v} \cdot \nabla \mathbf{w}=\left(c_{a}+c_{d}\right) \triangle \mathbf{w}+\left(c_{0}+c_{d}-c_{a}\right) \nabla(\nabla \cdot \mathbf{w})+2 \mu_{r}(\nabla \times \mathbf{v}-2 \mathbf{w}), \\
& \nabla \cdot \mathbf{v}=0 \\
& \eta_{e} \triangle \mathbf{H}=\nabla \times(\mathbf{H} \times \mathbf{v}), \\
& \nabla \cdot \mathbf{H}=0 \\
& \rho_{0} c_{v} \nabla T \cdot \mathbf{v}=k \triangle T \pm Q\left(T-T_{0}\right) \quad \text { in } \mathcal{S} .
\end{aligned}
$$

The sign + in front of $Q$ denotes heat generation whereas the sign - represents heat absorption.

All the material parameters are positive constants and $\mu_{e}$ is equal to the magnetic permeability of free space. As it is usual in the Boussinesq approximation $([13])$, in equation $(2)_{6}$ the dissipative terms have been neglected. 
We search $\mathbf{v}, \mathbf{w}, \mathbf{H}, T$ in the following form:

$$
\mathbf{v}=v_{1}\left(x_{2}\right) \mathbf{e}_{1}, \quad \mathbf{w}=w_{3}\left(x_{2}\right) \mathbf{e}_{3}, \quad \mathbf{H}=H_{0} \mathbf{e}_{2}+H_{1}\left(x_{2}\right) \mathbf{e}_{1}, \quad T=T\left(x_{2}\right),
$$

where $\left(\mathbf{e}_{1}, \mathbf{e}_{2}, \mathbf{e}_{3}\right)$ is the canonical base of $\mathbb{R}^{3}$. Thank to $(3), \mathbf{v}, \mathbf{w}, \mathbf{H}$ are divergence free.

The previous unknown functions satisfy the boundary conditions

$$
v_{1}( \pm d)=0, \quad w_{3}( \pm d)=0, \quad H_{1}( \pm d)=0, \quad T(-d)=T_{1}, \quad T(d)=T_{2} .
$$

We choice the reference temperature $T_{0}=\frac{T_{1}+T_{2}}{2}$ and we assume $T_{2}>T_{1}$.

By virtue of $(3)$ and $(2)_{1}$, we deduce $P=P\left(x_{1}\right)=-C x_{1}+p_{0}\left(C, p_{0}\right.$ some constants). By putting

$$
\begin{aligned}
& N^{2}=\frac{\mu_{r}}{\mu+\mu_{r}}, \quad l^{2}=\frac{c_{d}+c_{a}}{4 \mu}, \quad L=\frac{d}{l}, \quad M_{p}^{2}=N^{2} L^{2}, \quad M^{2}=\frac{\sigma_{e}}{\mu} \mu_{e}^{2} H_{0}^{2} d^{2}, \\
& V_{0}=\frac{C d^{2}}{\mu}, \quad \nu_{0}=\frac{\mu+\mu_{r}}{\rho_{0}}, \quad R e=\frac{V_{0} d}{\nu_{0}}, \\
& G r=\frac{\alpha_{T} g\left(T_{2}-T_{1}\right) d^{3}}{\nu_{0}^{2}}, \quad \lambda=\frac{G r}{R e}, \quad y=\frac{x_{2}}{d}, \\
& v(y)=\frac{v_{1}(d y)}{V_{0}}, \quad w(y)=\frac{d w_{3}(d y)}{V_{0}}, \quad \vartheta(y)=\frac{T(d y)-T_{0}}{T_{2}-T_{1}}, \quad h(y)=\frac{H_{1}(d y)}{V_{0} \sqrt{\sigma_{e} \mu}}, \\
& r^{2}=\frac{Q d^{2}}{k},
\end{aligned}
$$

equations (2) written in dimensionless form reduce to:

$$
\begin{aligned}
& v^{\prime \prime}+2 N^{2} w^{\prime}+M\left(1-N^{2}\right) h^{\prime}+\lambda \vartheta+1-N^{2}=0 \\
& w^{\prime \prime}-\frac{M_{p}^{2}}{2\left(1-N^{2}\right)} v^{\prime}-\frac{M_{p}^{2}}{1-N^{2}} w=0 \\
& h^{\prime \prime}+M v^{\prime}=0, \\
& \vartheta^{\prime \prime} \pm r^{2} \vartheta=0 \quad \text { in }[-1,1] .
\end{aligned}
$$

We recall that the coupling number $N^{2}$ belongs to $(0,1)$.

Boundary conditions (4) in dimensionless form become

$$
v( \pm 1)=0, \quad w( \pm 1)=0, \quad h( \pm 1)=0, \quad \vartheta( \pm 1)= \pm \frac{1}{2} .
$$




\section{Flow in the Case of Internal Heat Source}

We begin by considering the case of an internal heat source, which means to have the sign + in $(6)_{4}$. The integration of this equation taking into account $(7)_{4}$ shows that the solution does not exist if $r$ takes the values $n \pi$ where $n=1,2, \ldots$ and that the solution is not unique if $r$ takes the values $(2 n+1) \frac{\pi}{2}$ where $n=0,1, \ldots$. Otherwise the unique solution is given by:

$$
\vartheta(y)=\frac{\sin (r y)}{2 \sin r} \quad \text { in }[-1,1] .
$$

On taking into account the values of $r$ which make not well-set the problem $(6)_{4},(7)_{4}$, we will only consider $r \in(0, \pi) \backslash\{\pi / 2\}$.

In order to determine the other unknown functions, we begin by differentiating $(6)_{1,2}$. By virtue of $(6)_{3}$ and (8), $w$ has to satisfy the following linear ordinary differential equation:

$$
w^{I V}-\alpha w^{\prime \prime}+\beta w=\frac{\gamma r}{\sin r} \cos (r y),
$$

where

$$
\alpha=M_{p}^{2}+\left(1-N^{2}\right) M^{2}, \quad \beta=M_{p}^{2} M^{2}, \quad \gamma=-\frac{\lambda M_{p}^{2}}{4\left(1-N^{2}\right)} .
$$

The general solution of equation (9) depends on the sign of the discriminant $\Delta=\alpha^{2}-4 \beta$ of the algebraic equation

$$
\xi^{4}-\alpha \xi^{2}+\beta=0 .
$$

Therefore we solve problem (6), (7) in the three cases: $\Delta>0, \Delta=0, \Delta<0$.

These cases are all possible from the physical point of view because they represent the following situations:

- if $\Delta>0$, then $0<H_{0}<\sqrt{\frac{\mu}{\sigma_{e}}} \frac{M_{p}}{\mu_{e} d(1+N)}$ or $H_{0}>\sqrt{\frac{\mu}{\sigma_{e}}} \frac{M_{p}}{\mu_{e} d(1-N)}$ weak or strong external uniform magnetic field;

- if $\Delta=0$, then $H_{0}=\sqrt{\frac{\mu}{\sigma_{e}}} \frac{M_{p}}{\mu_{e} d(1+N)}$ or $H_{0}=\sqrt{\frac{\mu}{\sigma_{e}}} \frac{M_{p}}{\mu_{e} d(1-N)}$ critical external uniform magnetic field;

- if $\Delta<0$, then $\sqrt{\frac{\mu}{\sigma_{e}}} \frac{M_{p}}{\mu_{e} d(1+N)}<H_{0}<\sqrt{\frac{\mu}{\sigma_{e}}} \frac{M_{p}}{\mu_{e} d(1-N)}$ bounded external uniform magnetic field. 
Independently on the strength of the external magnetic field, a particular solution $w^{*}$ of $(9)$ is

$$
w^{*}(y)=\frac{\gamma r}{R \sin r} \cos (r y), \quad R=r^{4}+\alpha r^{2}+\beta
$$

so that the general solution of (9) is given by

$$
w(y)=w_{0}(y)+\frac{\gamma r}{R \sin r} \cos (r y)
$$

where $w_{0}(y)$ is the general solution of the homogeneous equation associate to (9) whose expression depends on the strength of the external magnetic field.

\section{1. $\Delta>0$ : Weak or Strong External Uniform Magnetic Field}

In this situation equation (11) admits the following real routes

$$
\begin{aligned}
& \xi_{1}=\sqrt{\frac{\alpha-\sqrt{\Delta}}{2}}=\frac{1}{2}\left[\sqrt{\left(M_{p}+M\right)^{2}-N^{2} M^{2}}-\sqrt{\left(M_{p}-M\right)^{2}-N^{2} M^{2}}\right], \\
& \xi_{2}=\sqrt{\frac{\alpha+\sqrt{\Delta}}{2}}=\frac{1}{2}\left[\sqrt{\left(M_{p}+M\right)^{2}-N^{2} M^{2}}+\sqrt{\left(M_{p}-M\right)^{2}-N^{2} M^{2}}\right], \\
& \xi_{3}=-\xi_{1}, \quad \xi_{4}=-\xi_{2}, .
\end{aligned}
$$

After some calculations we find that the solution of problem $(6)_{1,2,3},(7)_{1,2,3}$ is given by

$$
\begin{aligned}
v(y)= & \frac{M_{p}^{2}}{\xi_{2}^{2}-\xi_{1}^{2}} \frac{A_{1}^{+} \sinh \xi_{2}\left[\cosh \left(\xi_{1} y\right)-\cosh \xi_{1}\right]-A_{2}^{+} \sinh \xi_{1}\left[\cosh \left(\xi_{2} y\right)-\cosh \xi_{2}\right]}{\sinh \xi_{1} \sinh \xi_{2}} \\
& +\frac{2 \gamma}{R} \frac{A_{1}^{+} D_{2}^{+} \sinh \left(\xi_{1} y\right)-A_{2}^{+} D_{1}^{+} \sinh \left(\xi_{2} y\right)}{A_{1}^{+} \sinh \xi_{1} \cosh \xi_{2}-A_{2}^{+} \sinh \xi_{2} \cosh \xi_{1}}-\frac{2 \gamma S}{R \sin r} \sin (r y), \\
w(y)= & \frac{M_{p}^{2}}{2\left(\xi_{2}^{2}-\xi_{1}^{2}\right)} \frac{\sinh \xi_{2} \sinh \left(\xi_{1} y\right)-\sinh \xi_{1} \sinh \left(\xi_{2} y\right)}{\sinh \xi_{1} \sinh \xi_{2}} \\
& +\frac{\gamma}{R} \frac{D_{2}^{+} \cosh \left(\xi_{1} y\right)-D_{1}^{+} \cosh \left(\xi_{2} y\right)}{A_{1}^{+} \sinh \xi_{1} \cosh \xi_{2}-A_{2}^{+} \sinh \xi_{2} \cosh \xi_{1}}+\frac{\gamma r}{R \sin r} \cos (r y), \\
h(y)= & \frac{M_{p}^{2}}{M\left(\xi_{2}^{2}-\xi_{1}^{2}\right)} \frac{B_{1}^{+} \sinh \xi_{2} \sinh \left(\xi_{1} y\right)-B_{2}^{+} \sinh \xi_{1} \sinh \left(\xi_{2} y\right)}{\sinh \xi_{1} \sinh \xi_{2}}-\frac{y}{M}
\end{aligned}
$$




$$
\begin{aligned}
& +\frac{2}{M} \frac{\gamma}{R} \frac{B_{1}^{+} D_{2}^{+}\left[\cosh \left(\xi_{1} y\right)-\cosh \xi_{1}\right]-B_{2}^{+} D_{1}^{+}\left[\cosh \left(\xi_{2} y\right)-\cosh \xi_{2}\right]}{A_{1}^{+} \sinh \xi_{1} \cosh \xi_{2}-A_{2}^{+} \sinh \xi_{2} \cosh \xi_{1}} \\
& +\frac{2 \gamma M S}{R r \sin r}[\cos r-\cos (r y)] .
\end{aligned}
$$

where

$$
\begin{aligned}
& S=\frac{\left(1-N^{2}\right) r^{2}+M_{p}^{2}}{M_{p}^{2}}, \\
& A_{1}^{+}=\frac{1-N^{2}}{M_{p}^{2}} \xi_{1}-\frac{1}{\xi_{1}}, \quad A_{2}^{+}=\frac{1-N^{2}}{M_{p}^{2}} \xi_{2}-\frac{1}{\xi_{2}}, \quad B_{1}^{+}=1-\frac{\xi_{1}^{2}}{M_{p}^{2}}, \quad B_{2}^{+}=1-\frac{\xi_{2}^{2}}{M_{p}^{2}}, \\
& D_{1}^{+}=A_{1}^{+} r \cot r \sinh \xi_{1}+S \cosh \xi_{1}, \quad D_{2}^{+}=A_{2}^{+} r \cot r \sinh \xi_{2}+S \cosh \xi_{2} .
\end{aligned}
$$

\section{2. $\Delta=0$ : Critical External Uniform Magnetic Field}

In this case, equation (11) admits the following real routes

$$
\xi_{1}=\xi_{2}=\sqrt{\frac{\alpha}{2}}=\sqrt{M_{p} M}=: \xi, \quad \xi_{3}=\xi_{4}=-\xi_{1}
$$

so that problem $(6)_{1,2,3},(7)_{1,2,3}$ has the following solution

$$
\begin{aligned}
v(y) & =\frac{M_{p}^{2}}{2 \xi} \frac{A_{1}^{0}[\cosh \xi \cosh (\xi y)-y \sinh \xi \sinh (\xi y)-1]+A_{2}^{0} \sinh \xi[\cosh \xi-\cosh (\xi y)]}{\sinh ^{2} \xi} \\
& +\frac{2 \gamma}{R} \frac{\left(S A_{2}^{0} \cosh \xi-A_{1}^{0} D_{2}^{0}\right) \sinh (\xi y)+A_{1}^{0} D_{1}^{0} y \cosh (\xi y)}{A_{1}^{0}+A_{2}^{0} \sinh \xi \cosh \xi}-\frac{2 \gamma S}{R \sin r} \sin (r y), \\
w(y) & =\frac{M_{p}^{2}}{4 \xi} \frac{\cosh \xi \sinh (\xi y)-y \sinh \xi \cosh (\xi y)}{\sinh ^{2} \xi} \\
& +\frac{\gamma}{R} \frac{D_{1}^{0} y \sinh (\xi y)-\left(A_{2}^{0} r \cot r \sinh \xi+D_{2}^{0}\right) \cosh (\xi y)}{A_{1}^{0}+A_{2}^{0} \sinh \xi \cosh \xi}+\frac{\gamma r}{R \sin r} \cos (r y), \\
h(y) & =\frac{M_{p}^{2}}{2 \xi M} \frac{\left(B_{1}^{0} \cosh \xi+2 B_{2}^{0} \sinh \xi\right) \sinh (\xi y)-B_{1}^{0} y \sinh \xi \cosh (\xi y)}{\sinh { }^{2} \xi}-\frac{y}{M} \\
& +\frac{2 \gamma}{M R}\left\{B_{1}^{0}\left[r \cot r+\frac{D_{1}^{0} y \sinh (\xi y)-\left(A_{2}^{0} r \cot r \sinh \xi+D_{2}^{0}\right) \cosh (\xi y)}{A_{1}^{0}+A_{2}^{0} \sinh \xi \cosh \xi}\right]\right.
\end{aligned}
$$




$$
\left.+2 B_{2}^{0} \frac{D_{1}^{0}[\cosh \xi-\cosh (\xi y)]}{A_{1}^{0}+A_{2}^{0} \sinh \xi \cosh \xi}\right\}+\frac{2 \gamma M S}{R r \sin r}[\cos r-\cos (r y)]
$$

where

$$
\begin{aligned}
& A_{1}^{0}=\frac{1-N^{2}}{M_{p}^{2}} \xi-\frac{1}{\xi}, \quad A_{2}^{0}=\frac{1-N^{2}}{M_{p}^{2}}+\frac{1}{\xi^{2}}, \quad B_{1}^{0}=1-\frac{\xi^{2}}{M_{p}^{2}}, \quad B_{2}^{0}=\frac{\xi}{M_{p}^{2}}, \\
& D_{1}^{0}=A_{1}^{0} r \cot r \sinh \xi+S \cosh \xi, \quad D_{2}^{0}=A_{1}^{0} r \cot r \cosh \xi+S \sinh \xi
\end{aligned}
$$

\section{3. $\Delta<0$ : Bounded External Uniform Magnetic Field}

Equation (11) admits the following complex routes

$$
\begin{aligned}
& \xi_{1}=\frac{1}{2}\left[\sqrt{\left(M_{p}+M\right)^{2}-N^{2} M^{2}}-i \sqrt{N^{2} M^{2}-\left(M_{p}-M\right)^{2}}\right], \\
& \xi_{2}=\frac{1}{2}\left[\sqrt{\left(M_{p}+M\right)^{2}-N^{2} M^{2}}+i \sqrt{N^{2} M^{2}-\left(M_{p}-M\right)^{2}}\right], \\
& \xi_{3}=-\xi_{1}, \quad \xi_{4}=-\xi_{2} .
\end{aligned}
$$

Putting

$$
\begin{aligned}
& \delta=\frac{1}{2} \sqrt{\left(M_{p}+M\right)^{2}-N^{2} M^{2}}, \quad \sigma=\frac{1}{2} \sqrt{N^{2} M^{2}-\left(M_{p}-M\right)^{2}} \\
& A_{1}^{-}=\left(\frac{1-N^{2}}{M_{p}^{2}}-\frac{1}{M_{p} M}\right) \delta, \quad A_{2}^{-}=\left(\frac{1-N^{2}}{M_{p}^{2}}+\frac{1}{M_{p} M}\right) \sigma \\
& B_{1}^{-}=\frac{M_{p}^{2}-M^{2}\left(1-N^{2}\right)}{2 M_{p}^{2}}, \quad B_{2}^{-}=-\frac{2}{M_{p}^{2}} \sigma \delta \\
& D_{1}^{-}=r \cot r\left(A_{1}^{-} \cos \sigma \sinh \delta-A_{2}^{-} \sin \sigma \cosh \delta\right)+S \cos \sigma \cosh \delta \\
& D_{2}^{-}=r \cot r\left(A_{1}^{-} \sin \sigma \cosh \delta+A_{2}^{-} \cos \sigma \sinh \delta\right)+S \sin \sigma \sinh \delta
\end{aligned}
$$

the solution of the problem is given by:

$$
\begin{aligned}
v(y) & =\frac{M_{p}^{2}}{2 \delta \sigma\left(\sin ^{2} \sigma+\sinh ^{2} \delta\right)}\left[\left(A_{1}^{-} \sin \sigma \cosh \delta-A_{2}^{-} \cos \sigma \sinh \delta\right) \cos (\sigma y) \cosh (\delta y)\right. \\
& -\left(A_{1}^{-} \cos \sigma \sinh \delta+A_{2}^{-} \sin \sigma \cosh \delta\right) \sin (\sigma y) \sinh (\delta y)+A_{2}^{-} \sinh \delta \cosh \delta
\end{aligned}
$$




$$
\begin{aligned}
& \left.-A_{1}^{-} \cos \sigma \sin \sigma\right]+\frac{2 \gamma}{R} \\
& \times \frac{\left(A_{2}^{-} D_{1}^{-}-A_{1}^{-} D_{2}^{-}\right) \cos (\sigma y) \sinh (\delta y)+\left(A_{1}^{-} D_{1}^{-}+A_{2}^{-} D_{2}^{-}\right) \sin (\sigma y) \cosh (\delta y)}{A_{1}^{-} \sin \sigma \cos \sigma+A_{2}^{-} \sinh \delta \cosh \delta} \\
& -\frac{2 \gamma S}{R \sin r} \sin (r y), \\
w(y) & =\frac{M_{p}^{2}}{4 \delta \sigma} \frac{\sin \sigma \cosh \delta \cos (\sigma y) \sinh (\delta y)-\cos \sigma \sinh \delta \sin (\sigma y) \cosh (\delta y)}{\sin ^{2} \sigma+\sinh ^{2} \delta} \\
& +\frac{\gamma}{R} \frac{D_{1}^{-} \sin (\sigma y) \sinh (\delta y)-D_{2}^{-} \cos (\sigma y) \cosh (\delta y)}{A_{1}^{-} \sin \sigma \cos \sigma+A_{2}^{-} \sinh \delta \cosh \delta}+\frac{\gamma r}{R \sin r} \cos (r y), \\
h(y) & =\frac{M_{p}^{2}}{2 M \delta \sigma\left(\sin { }^{2} \sigma+\sinh { }^{2} \delta\right)}\left[\left(B_{1}^{-} \sin \sigma \cosh \delta-B_{2}^{-} \cos \sigma \sinh \delta\right)\right. \\
& \times \cos (\sigma y) \sinh (\delta y) \\
& \left.-\left(B_{1}^{-} \cos \sigma \sinh \delta+B_{2}^{-} \sin \sigma \cosh \delta\right) \sin (\sigma y) \cosh (\delta y)\right]-\frac{y}{M} \\
& +\frac{2 \gamma}{M R\left(A_{1}^{-} \sin \sigma \cos \sigma+A_{2}^{-} \sinh \delta \cosh \delta\right)}\{ \\
& +\left(B_{2}^{-} D_{1}^{-}-B_{1}^{-} D_{2}^{-}\right)[\cos (\sigma y) \cosh (\delta y)-\cos \sigma \cosh \delta] \\
& \left.\left.+D_{1}^{-}+B_{2}^{-} D_{2}^{-}\right)[\sin (\sigma y) \sinh (\delta y)-\sin \sigma \sinh \delta]\right\} \\
& 2 \gamma M)
\end{aligned}
$$

\section{Flow in the Case of Internal Heat Sink}

Now we suppose that there is an internal heat sink in the channel so that we have a sign - in $(6)_{4}$. The integration of this equation taking into account $(7)_{4}$ furnishes:

$$
\vartheta(y)=\frac{\sinh (r y)}{2 \sinh r} \quad \text { in }[-1,1]
$$


In this case equation (9) becomes:

$$
w^{I V}-\alpha w^{\prime \prime}+\beta w=\frac{\gamma r}{\sinh r} \cosh (r y)
$$

The general solution of equation (24) depends on the sign of $\Delta$; it is given by

$$
w(y)=w_{0}(y)+w^{*}(y)
$$

where $w_{0}(y)$ is the general solution of the homogeneous equation associate to (24) and the particular solution $w^{*}(y)$ is given by

$$
\begin{aligned}
& w^{*}(y)=\frac{\gamma r}{R \sinh r} \cosh (r y), \quad \text { if } r \text { is not root of }(11)(\Delta \gtreqless 0), \\
& w^{*}(y)=\frac{\gamma}{2\left(2 r^{2}-\alpha\right) \sinh r} y \sinh (r y), \quad \text { if } r \text { is a simple root of }(11)(\Delta>0), \\
& w^{*}(y)=\frac{\gamma r}{2\left(6 r^{2}-\alpha\right) \sinh r} y^{2} \cosh (r y), \quad \text { if } r \text { is double root of }(11)(\Delta=0),
\end{aligned}
$$

where in this Section

$$
R=r^{4}-\alpha r^{2}+\beta
$$

We now furnish the solution of the problem by using the expressions in (15), (18), (22) with suitable replacements. We recall that now we have to take into account two more cases. For the sake of brevity, we omit the calculations.

\section{1. $\Delta>0$ : Weak or Strong External Uniform Magnetic Field}

The roots of (11) are given by (14), but we have to distinguish two subcases.

\subsection{1. $r$ is not a Route of (11):}

The solution is obtained from (15) replacing the last term in the expressions of $v, w, h$ with

$$
+\frac{2 \gamma S}{R \sinh r} \sinh (r y),+\frac{\gamma r}{R \sinh r} \cosh (r y),+\frac{2 \gamma M S}{R r \sinh r}[\cosh r-\cosh (r y)],
$$

respectively, where now

$$
S=\frac{\left(1-N^{2}\right) r^{2}-M_{p}^{2}}{M_{p}^{2}}
$$

$$
D_{1}^{+}=A_{1}^{+} r \operatorname{coth} r \sinh \xi_{1}-S \cosh \xi_{1}, \quad D_{2}^{+}=A_{2}^{+} r \operatorname{coth} r \sinh \xi_{2}-S \cosh \xi_{2} .
$$




\subsection{2. $r$ is a Simple Route of (11):}

In this subcase, we hence have

$$
r=\xi_{i}, \quad i=1,2, \quad 2 r^{2}-\alpha=(-1)^{i}\left(\xi_{2}^{2}-\xi_{1}^{2}\right) .
$$

After some calculations we get three expressions analogous to (15) where now

$$
\begin{gathered}
D_{1}^{+}=A_{1}^{+} \xi_{i}^{2} \sinh \xi_{1}-\left[A_{i}^{+} \xi_{i}+2+A_{i}^{+} \xi_{i}^{2} \operatorname{coth} \xi_{i}\right] \cosh \xi_{1}, \\
D_{2}^{+}=A_{2}^{+} \xi_{i}^{2} \sinh \xi_{2}-\left[A_{i}^{+} \xi_{i}+2+A_{i}^{+} \xi_{i}^{2} \operatorname{coth} \xi_{i}\right] \cosh \xi_{2},
\end{gathered}
$$

the constant $R$ need to be replaced with $2(-1)^{i}\left(\xi_{2}^{2}-\xi_{1}^{2}\right) \xi_{i}^{2}$, and the last term in $v, w, h$ with

$$
\begin{gathered}
+\frac{\gamma}{(-1)^{i}\left(\xi_{2}^{2}-\xi_{1}^{2}\right) \xi_{i} \sinh \xi_{i}}\left[\left(A_{i}^{+}+\frac{2}{\xi_{i}}\right) \sinh \left(\xi_{i} y\right)+A_{i}^{+} \xi_{i} y \cosh \left(\xi_{i} y\right)\right], \\
+\frac{\gamma}{2(-1)^{i}\left(\xi_{2}^{2}-\xi_{1}^{2}\right) \sinh \xi_{i}} y \sinh \left(\xi_{i} y\right), \\
+\frac{\gamma}{M(-1)^{i}\left(\xi_{2}^{2}-\xi_{1}^{2}\right) \xi_{i} \sinh \xi_{i}}\left\{-2 \frac{(-1)^{i}\left(\xi_{2}^{2}-\xi_{1}^{2}\right)-\xi_{i}^{2}}{M_{p}^{2}}\left[\cosh \xi_{i}-\cosh \left(\xi_{i} y\right)\right]\right. \\
\left.+B_{i}^{+} \xi_{i}\left[y \sinh \left(\xi_{i} y\right)-\sinh \xi_{i}\right]\right\},
\end{gathered}
$$

respectively.

\section{2. $\Delta=0$ : Critical External Uniform Magnetic Field}

In this situation, equation (11) admits the real routes given by (17).

We have to distinguish two subcases.

\subsection{1. $r$ is not a Route of (11):}

The solution is obtained from (18) replacing the last term in the expressions of $v, w, h$ with

$$
-\frac{2 \gamma S}{R \sinh r} \sinh (r y),+\frac{\gamma r}{R \sinh r} \cosh (r y),-\frac{2 \gamma M S}{R r \sinh r}[\cosh r-\cosh (r y)],
$$

respectively, cot $r$ with coth $r$ in $h$ and in $D_{1}^{0}, D_{2}^{0}$ given by (19), while now

$$
S=\frac{M_{p}^{2}-\left(1-N^{2}\right) r^{2}}{M_{p}^{2}} .
$$




\subsection{2. $r$ is a Double Route of (11):}

$$
r^{2}=\xi^{2}=\alpha / 2
$$

Putting

$$
\begin{aligned}
& D_{1}^{0}=A_{1}^{0} \operatorname{coth} \xi \sinh \xi-\left(2 A_{2}^{0} \operatorname{coth} \xi+A_{1}^{0}-\frac{2}{\xi^{3}}\right) \cosh \xi \\
& D_{2}^{0}=A_{1}^{0} \operatorname{coth} \xi \cosh \xi-\left(2 A_{2}^{0} \operatorname{coth} \xi+A_{1}^{0}-\frac{2}{\xi^{3}}\right) \sinh \xi
\end{aligned}
$$

the solution is obtained from (18) replacing in the expressions of $v, w, h$ the constant $R$ with $8 \xi$, $\cot r$ with $\operatorname{coth} r$ and the last term with

$$
\begin{array}{r}
+\frac{\gamma}{2 \xi \sinh \xi} A_{2}^{0} y \cosh (\xi y)+\frac{\gamma}{4 \xi \sinh \xi}\left(A_{1}^{0} y^{2}-\frac{2}{\xi^{3}}\right) \\
\\
+\frac{\gamma}{8 \xi \sinh \xi} y^{2} \cosh (\xi y), \\
+\frac{\gamma}{4 M M_{p}^{2} \xi \sinh \xi}\left\{4 \xi[\sinh \xi-y \sinh (\xi y)]+\left(\xi^{2}-M_{p}^{2}\right)\left[\cosh \xi-y^{2} \cosh (\xi y)\right]\right. \\
-6[\cosh \xi-\cosh (\xi y)]\},
\end{array}
$$

respectively, where

$$
S=-2 A_{2}^{0} \operatorname{coth} \xi-A_{1}^{0}+\frac{2}{\xi^{3}}
$$

\section{3. $\Delta<0$ : Bounded External Uniform Magnetic Field}

In this situation, equation (11) admits the complex routes given by (20). After some calculations we deduce that $v, w, h$ have the expressions given by (22) substituting $\cot r$ with $\operatorname{coth} r$ and the last term with

$$
-\frac{2 \gamma S}{R \sinh r} \sinh (r y),+\frac{\gamma r}{R \sinh r} \cosh (r y),-\frac{2 \gamma M S}{R r \sinh r}[\cosh r-\cosh (r y)]
$$

respectively where $S$ is given by (27). 


\section{Comments on the Flow}

We make some comments on the flow both in the case of internal heat generation both in the case of internal heat absorption.

- If $r \ll 1$, then the solution tends to that one of the same problem in the absence of heat source or sink (see[1]).

- It is easy to verify that the electric field $\mathbf{E}$ is given by $E_{0} \mathbf{e}_{3}$ where $E_{0}$ is a constant independent of $\lambda$ and $r$, whose expression is given in [1]. Moreover outside the planes where there is a vacuum we have $\mathbf{E}=E_{0} \mathbf{e}_{3}, \mathbf{H}=$ $H_{0} \mathbf{e}_{2}$, by virtue of the usual transmission conditions for the electromagnetic field across $\Pi_{1,2}$.

- The Nusselt number at $\Pi_{1,2}$ defined by

$$
\mathrm{Nu}_{1,2}=\frac{k_{1,2} d}{k}=\left.\frac{d}{T_{2}-T_{1}} \frac{d T}{d x_{2}}\right|_{x_{2}= \pm d}=\vartheta^{\prime}( \pm 1),
$$

has the following form

$$
\begin{aligned}
& \mathrm{Nu}_{1,2}=\frac{r}{2} \cot r, \text { if there is a source, } \\
& \mathrm{Nu}_{1,2}=\frac{r}{2} \operatorname{coth} r, \text { if there is a sink. }
\end{aligned}
$$

If $r$ tends to $0^{+}$then $\mathrm{Nu}_{1,2}$ tends to $1 / 2$ according to [1].

The heat flux vector at the walls in the case of heat generation is given by

$$
\mathbf{q}=-\frac{\left(T_{2}-T_{1}\right) k r}{2 d} \cot r \mathbf{e}_{2},
$$

so that the sign of $N u_{1,2}$ and the direction of $\mathbf{q}$ depend on the values of $r$. In the case of heat absorption it is sufficient to replace $\cot r$ with $\operatorname{coth} r$ : the sign of $N u_{1,2}$ is positive and the direction of $\mathbf{q}$ is opposite of $\mathbf{e}_{2}$ as in the absence of source or sink ([1]).

- The skin friction $\left(\boldsymbol{\tau}_{1,2}\right)$ and the skin couple friction $\left(\boldsymbol{\tau}_{p 1,2}\right)$ at both plates are given by

$$
\boldsymbol{\tau}_{1,2}=\left(\mu+\mu_{r}\right) \frac{V_{0}}{d} v^{\prime}( \pm 1) \mathbf{e}_{1} ; \quad \boldsymbol{\tau}_{p 1,2}=\left(c_{d}+c_{a}\right) \frac{V_{0}}{d^{2}} w^{\prime}( \pm 1) \mathbf{e}_{3} .
$$

The expression of $\boldsymbol{\tau}_{1,2}$ is related to the occurrence of the reverse flow, as we will see in the next section. 
- If $C=0$, then the solution is obtained from the previous expressions writing only the terms having coefficient $\gamma$. Of course in the dimensionless variables, the reference velocity $V_{0}$ cannot be expressed in terms of $C$.

- If $H_{0}=0$ and there is a source, then the motion is given by

$$
\begin{aligned}
v(y)= & \frac{1}{2}\left(1-y^{2}\right)+\frac{N^{2}\left[\cosh \left(M_{p} y\right)-\cosh M_{p}\right]}{M_{p} \sinh M_{p}} \\
& -\frac{\lambda N^{2} M_{p}^{2} \cot r\left[\sinh \left(M_{p} y\right)-y \sinh M_{p}\right]}{2 r\left(1-N^{2}\right)\left(r^{2}+M_{p}^{2}\right)\left(M_{p} \cosh M_{p}-N^{2} \sinh M_{p}\right)} \\
& +\frac{\lambda\left[M_{p}^{2}+\left(1-N^{2}\right) r^{2}\right]}{2 r^{2}\left(r^{2}+M_{p}^{2}\right)\left(1-N^{2}\right)} \\
& \times\left\{\frac{\sin (r y)}{\sin r}-\frac{y M_{p} \cosh M_{p}-N^{2} \sinh \left(M_{p} y\right)}{M_{p} \cosh M_{p}-N^{2} \sinh M_{p}}\right\}, \\
w(y)= & \frac{1}{2}\left[y-\frac{\sinh \left(M_{p} y\right)}{\left.\sinh M_{p}\right]}\right. \\
& -\frac{\lambda M_{p}\left[M_{p}^{2}+\left(1-N^{2}\right) r^{2}\right]\left[\cosh \left(M_{p} y\right)-\cosh M_{p}\right]}{4 r^{2}\left(1-N^{2}\right)\left(r^{2}+M_{p}^{2}\right)\left(M_{p} \cosh M_{p}-N^{2} \sinh M_{p}\right)} \\
& -\frac{\lambda M_{p}^{2}}{4 r^{2}\left(r^{2}+M_{p}^{2}\right)\left(1-N^{2}\right)} \\
& \times\left\{r \frac{\cos (r y)}{\sin r}-\frac{r \cot r\left[M_{p} \cosh \left(M_{p} y\right)-N^{2} \sinh M_{p}\right]}{M_{p} \cosh M_{p}-N^{2} \sinh M_{p}}\right\} .
\end{aligned}
$$

If there is a sink, then we have to consider two cases according to the value of $r$.

If $r \neq M_{p}$, then the motion is obtained from (29) by replacing the trigonometric functions $\sin , \cos$, cot with the hyperbolic functions sinh, cosh, coth, the constant $r^{2}+M_{p}^{2}$ with $r^{2}-M_{p}^{2}$ and $M_{p}^{2}+\left(1-N^{2}\right) r^{2}$ with $M_{p}^{2}-(1-$ $\left.N^{2}\right) r^{2}$.

If $r=M_{p}$, then

$$
\begin{aligned}
v(y)= & \frac{1}{2}\left(1-y^{2}\right)+\frac{N^{2}\left[\cosh \left(M_{p} y\right)-\cosh M_{p}\right]}{M_{p} \sinh M_{p}} \\
& +\frac{\lambda N^{2}}{4 M_{p}\left(1-N^{2}\right) \sinh M_{p}}\left[y \cosh \left(M_{p} y\right)\right.
\end{aligned}
$$




$$
\begin{aligned}
& \left.-\frac{\left(M_{p} \sinh M_{p}-N^{2} \cosh M_{p}\right) \sinh \left(M_{p} y\right)-M_{p} y}{M_{p} \cosh M_{p}-N^{2} \sinh M_{p}}\right], \\
& -\frac{\lambda\left(2-N^{2}\right)}{4 M_{p}^{2}\left(1-N^{2}\right)}\left[\frac{\sinh \left(M_{p} y\right)}{\sinh M_{p}}-\frac{M_{p} y \cosh M_{p}-N^{2} \sinh \left(M_{p} y\right)}{M_{p} \cosh M_{p}-N^{2} \sinh M_{p}}\right], \\
& w(y)=\frac{1}{2}\left[y-\frac{\sinh \left(M_{p} y\right)}{\sinh M_{p}}\right] \\
& +\frac{\lambda\left[2-N^{2}-M_{p} N^{2} \operatorname{coth} M_{p}\right]\left[\cosh \left(M_{p} y\right)-\cosh M_{p}\right]}{8 M_{p}\left(1-N^{2}\right)\left(M_{p} \cosh M_{p}-N^{2} \sinh M_{p}\right)} \\
& -\frac{\lambda}{8\left(1-N^{2}\right)}\left[y \frac{\sinh \left(M_{p} y\right)}{\sinh M_{p}}-\frac{M_{p} \cosh \left(M_{p} y\right)-N^{2} \sinh M_{p}}{M_{p} \cosh M_{p}-N^{2} \sinh M_{p}}\right] .
\end{aligned}
$$

- If $\mathcal{S}$ is occupied by a Boussinesquian, electrically conducting Newtonian fluid, then its MHD mixed convective flow is governed by $(6)_{1}$ with $w=0$, $N=0,(6)_{3,4}$ and the boundary conditions $(7)_{1,3,4}$. The dimensionless temperature $\vartheta$ is given by (8) and

$$
\begin{aligned}
v(y)= & \frac{\cosh M-\cosh (M y)}{M \sinh M}+\frac{\lambda}{2\left(M^{2}+r^{2}\right)}\left[\frac{\sin (r y)}{\sin r}-\frac{\sinh (M y)}{\sinh M}\right], \\
h(y)= & \frac{1}{M}\left[\frac{\sinh (M y)}{\sinh M}-y\right]+\frac{\lambda[\cosh (M y)-\cosh M]}{2\left(M^{2}+r^{2}\right) \sinh M} \\
& +\frac{\lambda M[\cos (r y)-\cos r]}{2 r\left(M^{2}+r^{2}\right) \sin r}
\end{aligned}
$$

if we assume the presence of an internal heat source.

If there is an internal heat sink, then the dimensionless temperature $\vartheta$ is given by (23) and we have to distinguish two cases.

If $r \neq M$, then in the previous expressions of $v, h$ we have to replace the functions sin, cos with sinh, cosh, the constant $M^{2}+r^{2}$ with $M^{2}-r^{2}$ and we have to change the sign in the last term in $h$.

If $r=M$, then

$$
\begin{aligned}
v(y)= & \frac{\cosh M-\cosh (M y)}{M \sinh M} \\
& +\frac{\lambda}{4 M \sinh ^{2} M}[\cosh M \sinh (M y)-y \sinh M \cosh (M y)],
\end{aligned}
$$




$$
\begin{aligned}
h(y)= & \frac{1}{M}\left[\frac{\sinh (M y)}{\sinh M}-y\right]+\frac{\lambda(1+M \operatorname{coth} M)}{4 M^{2} \sinh M}[\cosh M-\cosh (M y)] \\
& +\frac{\lambda}{4 M}\left[y \frac{\sinh (M y)}{\sinh M}-1\right] .
\end{aligned}
$$

- In our study we have assumed $T_{2}>T_{1} ;$ if $T_{2}=T_{1}$ then it is easy to prove that $\vartheta=0$. Hence we obtain that the flow is not influenced by the internal heat source or sink.

Due to the geometry of the problem, all the previous relations hold for all $y \in[-1,1]$.

\section{Numerical Simulations and Discussions}

The aim of this Section is to present a selected set of graphical and numerical results illustrating the effects on the flow of the presence of an internal heat source or sink. This target can be obtained by studying the behavior of the flow when $r$ varies. Actually, the internal heat parameter $r^{2}(r \in(0, \pi) \backslash\{\pi / 2\}$ in the case of internal source) is related to the constant rate of volumetric internal heat generation.

As it is proved in the previous Sections, the solution of the problem depends also on the values of some other relevant physical dimensionless parameters which describe the micropolar properties of the fluid $\left(N\right.$ and $\left.M_{p}\right)$, the electromagnetic properties of the flow $\left(M^{2}\right)$ and the buoyancy forces due to the gravity $(\lambda)$. Nevertheless, when the value of $r$ is fixed, the influence of the other parameters on the flow is not modified in a relevant way by the presence of the source or sink, so that we refer to [1] for a complete study of the behavior of the motion when the parameters $N^{2}, M_{p}, M^{2}$ and $\lambda$ change. Briefly, the behavior of the micropolar flow differs highly from the Newtonian one as $N^{2}$ increases and $M_{p}$ decreases, the velocity and microrotation decrease as $M^{2}$ increases and for suitable vales of the buoyancy parameter $\lambda$ the reverse flow occurs as we will see below.

We provide Figures 2, 3 in order to show the influence of $r \in(0,3]$ on the temperature, velocity, microrotation and induced magnetic field. The graphics given for $r=0$ represent the flow in the absence of internal heat source or sink studied in [1].

As far as the temperature is concerned, the trend depends only on the parameter $r$ : if $0<r \leq 1$, it is approximatively linear. If there is a source then in the other cases $(1<r \leq 3)$ the temperature $\vartheta$ takes minimum and maximum 

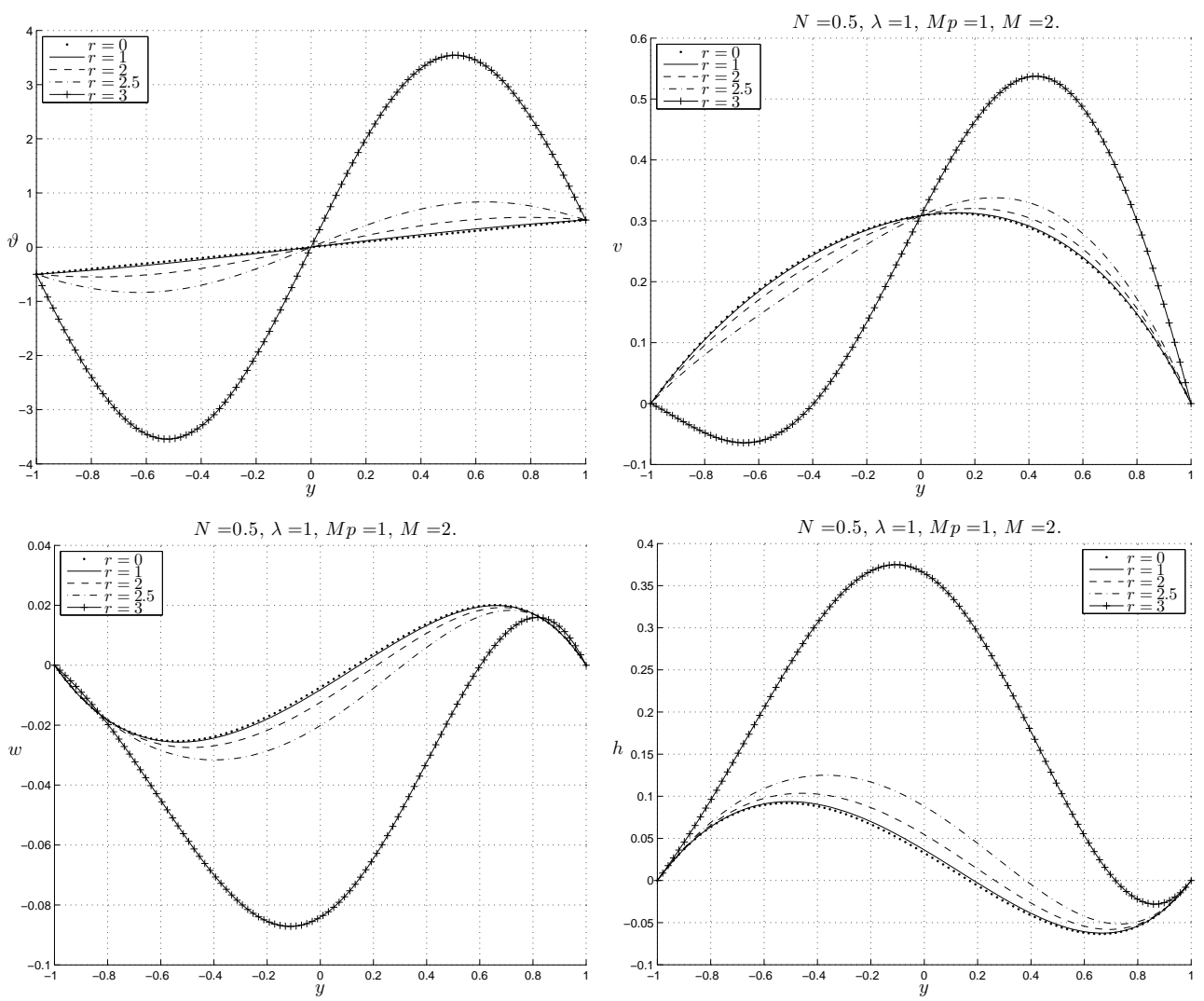

Figure 2: Heat source: the effect of $r$ on the temperature (which depends only on $r$ ), on the velocity, on the microrotation and on the induced magnetic field.

which are very accentuated for $r$ close to $\pi$; if there is a sink then $\vartheta$ is always an increasing function. For the other functions, in the case of source, if $r=3$ then the behavior is very different with respect to the other values and the reverse flow appears for the velocity. When an internal heat sink is present, the behavior of the velocity, the microrotation and the magnetic field is very slightly influenced by $r$.

The reverse flow appears when the dimensional velocity and the gradient of $P$ have the same direction. In our problem, we have that the gradient of $P$ $\left(\nabla P=-C \mathbf{e}_{1}\right)$ depends only on the constant $C$, which appears in the expression of the buoyancy parameter $\lambda$. Hence, in order to study the occurrence of the reverse flow, it is useful to provide Figure 4, where we can see the behavior 

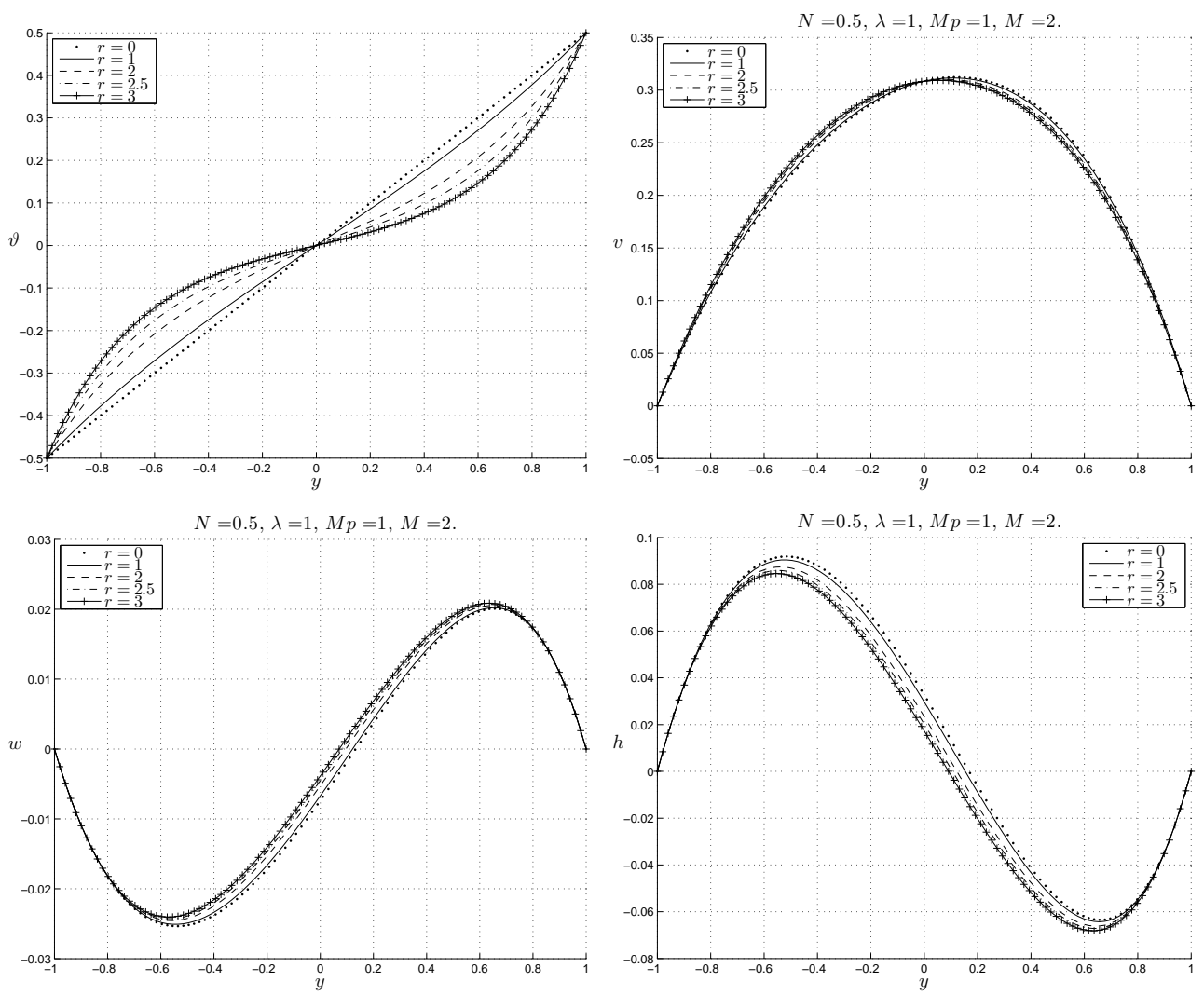

Figure 3: Heat sink: the effect of $r$ on the temperature (which depends only on $r$ ), on the velocity, on the microrotation and on the induced magnetic field.

of the velocity when $\lambda$ changes in the presence of a source or a sink.

If $\lambda=0$ (symmetric heating, i.e. $T_{1}=T_{2}$ ), then the pictures show that $v$ is always positive so that the dimensional velocity $\left(\mathbf{v}=v_{1} \mathbf{e}_{\mathbf{1}}\right)$ and the gradient of $P\left(\nabla P=-C \mathbf{e}_{\mathbf{1}}\right)$ have opposite direction, provided $C \neq 0$ ( different choices of the values of the other parameters do not modify the profile of the velocity). Hence, in the case $\lambda=0$ the reverse flow does not occur. Therefore, the occurrence of the reverse flow is a feature of the mixed convection in the case of asymmetric heating. Actually, there exists a critical value $\lambda^{*}$ of $\lambda$ such that

- $C>0(\Longleftrightarrow \lambda>0)$ : if $\lambda \leq \lambda^{*}$, then the reverse flow does not appear; if $\lambda>\lambda^{*}$, then the reverse flow occurs near the coldest wall; 

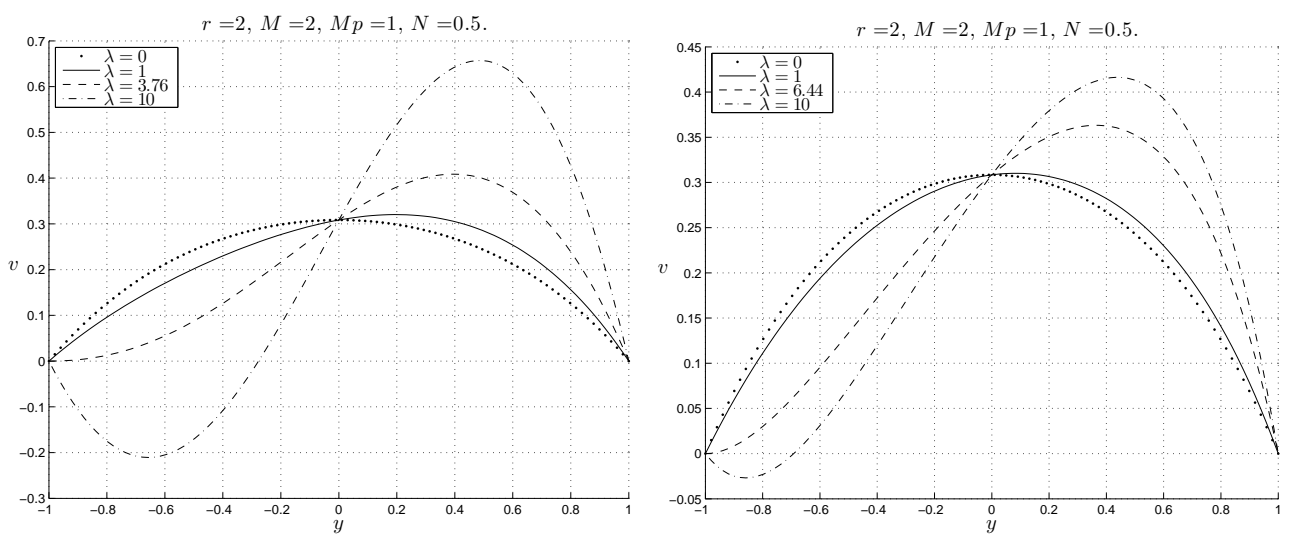

Figure 4: The effect of $\lambda$ on the velocity when an internal heat source (first picture) or sink (second picture) is present $(\Delta=0)$.

- $C<0(\Longleftrightarrow \lambda<0)$ : if $\lambda \geq \lambda^{*}$, then the reverse flow does not appear; if $\lambda<\lambda^{*}$, then the reverse flow occurs near the hottest wall.

The value of $\lambda^{*}$ depends on the other parameters and it is computed by putting $\boldsymbol{\tau}_{1,2}$ equal to zero. In the fifth and sixth columns of Table 1 the values of $\lambda_{\text {source }}^{*}$ and $\lambda_{\text {sink }}^{*}$ are reported when $r, M_{p}, N$, and $M$ change (first four columns) for $C>0$. From this Table we can easily obtain the corresponding critical values of $\lambda^{*}$ when $C<0$ because the profiles of $v$ for negative values of $\lambda$ can be found by symmetry from the corresponding graphics of $v$ when $\lambda>0$.

From the examination of Table 1 it appears that $\lambda^{*}$ is an increasing function in $M$, a decreasing function in $N$ and it is not influenced in a relevant way by $M_{p}$ as in [1]. Moreover $\lambda^{*}$ decreases as $r$ increases if there is a source (the decreasing is very significant when $r=3$ ), while $\lambda^{*}$ increases as $r$ increases if there is a sink. So the presence of a source tends to favor the occurrence of the reverse flow, while the presence of a sink tends to prevent the occurrence of the reverse flow. As far as we know, this phenomenon has not been pointed out in the literature.

As in other physical situations ([14], [1]), the influence of $M$ on $\lambda^{*}$ shows that the presence of the external magnetic field tends to prevent the occurrence of the reverse flow. For fixed values of $r$ and $M$, we have that $\lambda^{*}$ is smaller than in Newtonian case and when $N \rightarrow 1^{-} \lambda^{*}$ differs highly from the corresponding value in the Newtonian case (see Tables 1 and 2).

Finally we consider the behavior of the induced magnetic field, which is usually neglected in the literature. Figure 5 shows the influence of the strength 


\begin{tabular}{|c|c|c|c|c|c|c|c|c|}
\hline$r$ & $M_{p}$ & $N$ & $\bar{M}$ & $\lambda_{\text {source }}^{*}$ & $\lambda_{\operatorname{sink}}^{*}$ & $\lambda$ & $M_{\text {source }}^{*}$ & $M_{\text {sink }}^{*}$ \\
\hline \multirow[t]{12}{*}{1} & \multirow[t]{6}{*}{1} & \multirow[t]{3}{*}{0.20} & 1 & 5.6993 & 6.4941 & 1 & 3.2482 & 3.2883 \\
\hline & & & 2 & 6.6439 & 7.5021 & 5 & 3.1882 & 3.2082 \\
\hline & & & 10 & 20.3388 & 21.4946 & 10 & 3.2362 & 3.2442 \\
\hline & & \multirow[t]{3}{*}{0.90} & 1 & 0.9801 & 1.1253 & 1 & 6.3033 & 6.3033 \\
\hline & & & 2 & 1.0262 & 1.1751 & 5 & 6.5636 & 6.5836 \\
\hline & & & 10 & 2.0204 & 2.2175 & 10 & 6.5796 & 6.6036 \\
\hline & \multirow[t]{6}{*}{5} & \multirow[t]{3}{*}{0.20} & 1 & 5.6911 & 6.4848 & 1 & 3.2082 & 3.2482 \\
\hline & & & 2 & 6.6451 & 7.5025 & 5 & 3.1562 & 3.1722 \\
\hline & & & 10 & 20.3653 & 21.5173 & 10 & 3.2082 & 3.2122 \\
\hline & & \multirow[t]{3}{*}{0.90} & 1 & 0.9956 & 1.1402 & 1 & 3.8889 & 3.8529 \\
\hline & & & 2 & 1.0868 & 1.2372 & 5 & 4.2172 & 4.2252 \\
\hline & & & 10 & 2.4024 & 2.5832 & 10 & 4.2372 & 4.2533 \\
\hline \multirow[t]{12}{*}{2} & \multirow[t]{6}{*}{1} & \multirow[t]{3}{*}{0.20} & 1 & 4.2881 & 7.5354 & 1 & 3.1842 & 3.3283 \\
\hline & & & 2 & 5.0879 & 8.6107 & 5 & 3.1922 & 3.2402 \\
\hline & & & 10 & 17.9197 & 22.8685 & 10 & 3.2322 & 3.2603 \\
\hline & & \multirow[t]{3}{*}{0.90} & 1 & 0.7269 & 1.3178 & 1 & 6.3554 & 6.3353 \\
\hline & & & 2 & 0.7647 & 1.3719 & 5 & 6.5315 & 6.6076 \\
\hline & & & 10 & 1.6396 & 2.4624 & 10 & 6.5395 & 6.6396 \\
\hline & \multirow[t]{6}{*}{5} & \multirow[t]{3}{*}{0.20} & 1 & 4.2819 & 7.5247 & 1 & 3.1481 & 3.2883 \\
\hline & & & 2 & 5.0902 & 8.6099 & 5 & 3.1602 & 3.2082 \\
\hline & & & 10 & 17.9536 & 22.8867 & 10 & 3.2002 & 3.2322 \\
\hline & & \multirow[t]{3}{*}{0.90} & 1 & 0.7419 & 1.3315 & 1 & 3.9930 & 3.8248 \\
\hline & & & 2 & 0.8194 & 1.4342 & 5 & 4.2012 & 4.2292 \\
\hline & & & 10 & 2.0402 & 2.8040 & 10 & 4.2092 & 4.2773 \\
\hline \multirow[t]{12}{*}{3} & \multirow[t]{6}{*}{1} & \multirow[t]{3}{*}{0.20} & 1 & 0.8536 & 8.9956 & 1 & 3.1682 & 3.3283 \\
\hline & & & 2 & 1.0659 & 10.1415 & 5 & 3.2082 & 3.2963 \\
\hline & & & 10 & 6.5355 & 24.6235 & 10 & 3.2122 & 3.3043 \\
\hline & & \multirow[t]{3}{*}{0.90} & 1 & 0.1391 & 1.5917 & 1 & 6.4635 & 6.3594 \\
\hline & & & 2 & 0.1483 & 1.6505 & 5 & 6.4675 & 6.6476 \\
\hline & & & 10 & 0.4169 & 2.7877 & 10 & 6.4675 & 6.6957 \\
\hline & \multirow[t]{6}{*}{5} & \multirow[t]{3}{*}{0.20} & 1 & 0.8524 & 8.9830 & 1 & 3.1401 & 3.2883 \\
\hline & & & 2 & 1.0673 & 10.1388 & 5 & 3.1802 & 3.2603 \\
\hline & & & 10 & 6.5701 & 24.6362 & 10 & 3.1842 & 3.2723 \\
\hline & & \multirow[t]{3}{*}{0.90} & 1 & 0.1439 & 1.6025 & 1 & 4.1491 & 3.8208 \\
\hline & & & 2 & 0.1643 & 1.7105 & 5 & 4.1572 & 4.2292 \\
\hline & & & 10 & 0.6293 & 3.0944 & 10 & 4.1572 & 4.3093 \\
\hline
\end{tabular}

Table 1: Micropolar: Critical values $\lambda^{*}, M^{*}$ of $\lambda$ and $M$.

of the external magnetic field and the electromagnetic properties of the fluid on $h$ in the case of a source or a sink. From these pictures, it appears that the absolute value of $h$ is an increasing function in $M$ until $M$ reaches a critical 


\begin{tabular}{c|ccc|ccc}
\hline$r$ & $M$ & $\lambda_{\text {source }}^{*}$ & $\lambda_{\text {sink }}^{*}$ & $\lambda$ & $M_{\text {source }}^{*}$ & $M_{\text {sink }}^{*}$ \\
\hline 1 & 1 & 5.9618 & 6.7915 & 1 & 3.1855 & 3.2231 \\
& 2 & 6.9806 & 7.8782 & 5 & 3.1303 & 3.1404 \\
& 10 & 21.5860 & 22.7928 & 10 & 3.1729 & 3.1779 \\
2 & 1 & 4.4876 & 7.8782 & 1 & 3.1529 & 3.2657 \\
& 2 & 5.3513 & 9.0368 & 5 & 3.1253 & 3.1729 \\
& 10 & 19.0558 & 24.2260 & 10 & 3.1679 & 3.1955 \\
3 & 1 & 0.8945 & 9.4014 & 1 & 3.1078 & 3.2607 \\
& 2 & 1.1245 & 10.6351 & 5 & 3.1479 & 3.2281 \\
& 10 & 7.0219 & 26.0555 & 10 & 3.1504 & 3.2356 \\
\hline
\end{tabular}

Table 2: Newtonian: Critical values of $\lambda$ and $M$.
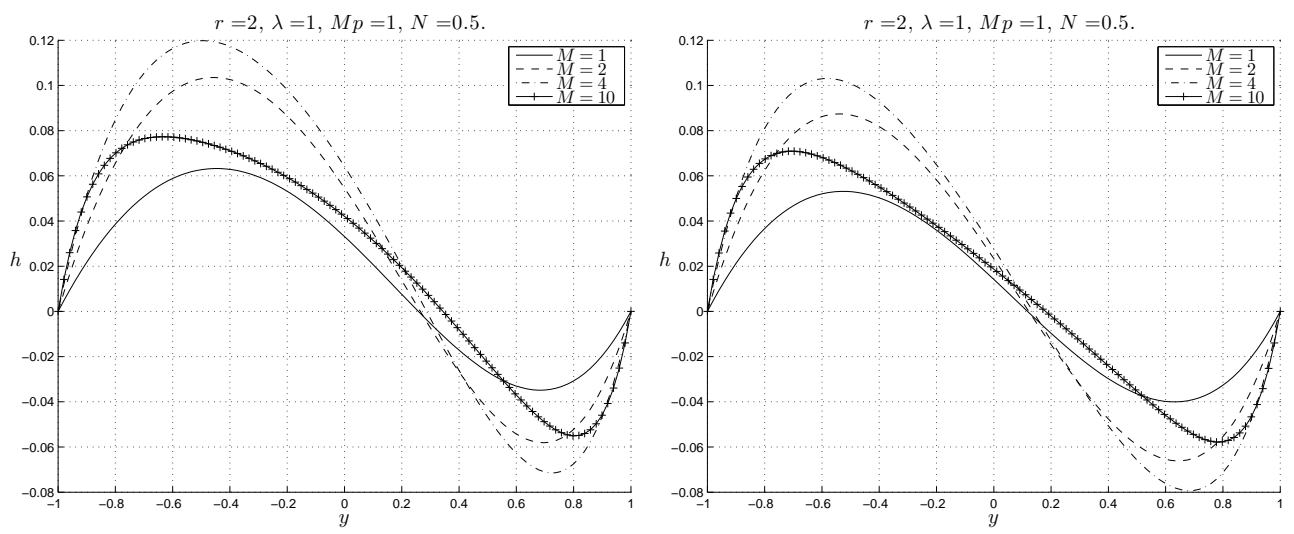

Figure 5: The effect of $M$ on the induced magnetic field in the case of a source or a sink, respectively. If $M=1,2,4,10$, then $\Delta<0, \Delta=$ $0, \Delta>0, \Delta>0$, respectively.

value $M^{*}$. If $M>M^{*}$, then the absolute value of $h$ becomes a decreasing function in $M$. This behavior of $h$ has been first emphasized in [1].

In the eighth and ninth columns of Table 1 we can read the values of $M_{\text {source }}^{*}$ and $M_{\text {sink }}^{*}$ when $r, M_{p}, N$, and $\lambda$ increase (first, second, third and seventh columns). We note that $M^{*}$ does not depend on $r$ in a relevant way.

Finally, it is interesting to compare these values with the corresponding value of $M^{*}$ in the Newtonian case (Table 2). In the micropolar fluid, $M^{*}$ is always grater than in the Newtonian fluid and its value increases as $N$ increases. This behavior can be expected because when $N \rightarrow 1^{-}$the fluid differs highly from the Newtonian one. 


\section{Acknowledgments}

Our research is partially supported by GNFM of Italian Istituto Nazionale di Alta Matematica.

\section{References}

[1] A. Borrelli, G. Giantesio, M.C. Patria, Magnetoconvection of a micropolar fluid in a vertical channel, Int. J. Heat and Mass Transfer, 80, (2015), 614-625, doi: 10.1016/j.ijheatmasstransfer.2014.09.031.

[2] K.R. Rajagopal, M. M. Ruzicka, A. R. Srinivasa, On the Oberbeck-Boussinesq approxiamtion, Mathematical Models and Methods in Applied Sciences, 6, No. 8 (1996), 11571167, doi: 10.1142/S0218202596000481.

[3] A.C. Eringen, Theory of micropolar fluids, J. Math. Mech., 16, No. 1 (1966), 1-18, doi: 10.1512/iumj.1967.16.16001.

[4] A.C. Eringen, Microcontinuum Field Theories, Vol. I - II, Springer-Verlag (2001).

[5] G. Lukaszewicz, Micropolar Fluids Theory and Applications, Birkäuser (1999).

[6] K. Vajravelu, A. Hadjinicalaou, Heat transfer in a viscous fluid over a stretching sheet with viscous dissipation and internal heat generation, Int. Comm. Heat Mass Transfer, 20, No. 3 (1993), 417-430, doi: 10.1016/0735-1933(93)90026-R.

[7] M.M. Rahman, M.A. Sattar, Magnetohydrodynamic convective flow of a micropolar fluid past a continuously moving vertical porous plate in the presence of heat generation/absorption, ASME J. Heat Transfer, 128, No. 2 (2006), 142-152, doi: 10.1115/1.2136918.

[8] E. Magyari, I. Pop. A. Postelnicu, Effect of the source therm on steady free convection boundary layer flows over a vertical plate in a porous medium. Part I, Transport in porous media, 67, No. 1 (2007), 49-67, doi: 10.1007/s11242-006-0012-1.

[9] A.J. Chamkha, T. Grosan, I. Pop, Fully developed free convection of a micropolar fluid in a vertical channel, Int. Comm. Heat Mass Transfer, 29, No. 8 (2002), 1119-1127, doi: 10.1016/S0735-1933(02)00440-2.

[10] C.Y. Cheng, Fully developed natural convection heat and mass transfer of a micropolar fluid in a vertical channel with asymmetric wall temperatures and concentrations, Int. Comm. Heat Mass Transfer, 33, No. 5 (2006), 627-635, doi: 10.1016/j.icheatmasstransfer.2006.01.014.

[11] J.C. Umavathi, J. Sultana, Mixed convection flow of a micropolar fluid in a vertical channel with boundary conditions of third kind, Int. J. Eng. Sci. Tech., 3, No. 4 (2011), 213-224, doi: 10.4314/ijest.v3i4.68554.

[12] J.C. Umavathi, J. Sultana, Mixed convection flow of a micropolar fluid with concentration in a vertical channel in the presence of heat source or sink, IJMA, 3, No. 10 (2012), 35563569 . 
[13] S. Chandrasekhar, Hydrodynamic and hydromagnetic stability, Dover Publications, Inc. New York (1981).

[14] A. Borrelli, G. Giantesio, M.C. Patria, Numerical simulations of three-dimensional MHD stagnation-point flow of a micropolar fluid, CAMWA, 66, No. 4 (2013), 472-489, doi: 10.1016/j.camwa.2013.05.023.

[15] A. Barletta, Laminar mixed convection with viscous dissipation in a vertical channel, Int. J. Heat and Mass Transfer, 41, No. 22 (1998), 3501-3513, doi: 10.1016/S00179310(98)00074-X. 